\title{
Partial-Wave Representations of Laser Beams for Use in Light- Scattering Calculations
}

Gérard Gouesbet

James A. Lock

Cleveland State University, j.lock@csuohio.edu

Gérard Gréhan

Follow this and additional works at: https://engagedscholarship.csuohio.edu/sciphysics_facpub

Part of the Physics Commons

How does access to this work benefit you? Let us know!

\section{Publisher's Statement}

This paper was published in Applied Optics and is made available as an electronic reprint with the permission of OSA. The paper can be found at the following URL on the OSA website: http://www.opticsinfobase.org/ao/abstract.cfm?URI=ao-34-12-2133. Systematic or multiple reproduction or distribution to multiple locations via electronic or other means is prohibited and is subject to penalties under law.

\section{Original Citation}

Gouesbet, Gérard, James A. Lock, and Gérard Gréhan. "Partial-Wave Representations of Laser Beams for Use in Light-Scattering Calculations." Applied Optics 34 (1995): 2133-2143.

\section{Repository Citation}

Gouesbet, Gérard; Lock, James A.; and Gréhan, Gérard, "Partial-Wave Representations of Laser Beams for Use in Light-Scattering Calculations" (1995). Physics Faculty Publications. 11.

https://engagedscholarship.csuohio.edu/sciphysics_facpub/11

This Article is brought to you for free and open access by the Physics Department at EngagedScholarship@CSU. It has been accepted for inclusion in Physics Faculty Publications by an authorized administrator of EngagedScholarship@CSU. For more information, please contact library.es@csuohio.edu. 


\title{
Partial-wave representations of laser beams for use in light-scattering calculations
}

\author{
Gérard Gouesbet, James A. Lock, and Gérard Gréhan
}

\begin{abstract}
In the framework of generalized Lorenz-Mie theory, laser beams are described by sets of beam-shape coefficients. The modified localized approximation to evaluate these coefficients for a focused Gaussian beam is presented. A new description of Gaussian beams, called standard beams, is introduced. A comparison is made between the values of the beam-shape coefficients in the framework of the localized approximation and the beam-shape coefficients of standard beams. This comparison leads to new insights concerning the electromagnetic description of laser beams. The relevance of our discussion is enhanced by a demonstration that the localized approximation provides a very satisfactory description of top-hat beams as well.
\end{abstract}

\section{Introduction}

Many optical particle-sizing techniques rely on the interaction between laser beams and the particles that are being studied. Examples are provided by Gaussian laser beams used in phase-Doppler instruments, ${ }^{1-3}$ laser sheets used in particle-image vel ocimetry, ${ }^{4-6}$ and top-hat beams used in the so-called tophat technique. 7-10 If the diameter of the spherical particles that are being studied is comparable to the characteristic beam width, the theoretical analysis of the light-scattering signature of the particles must rely on generalized Lorenz-Mie theory (GLMT) rather than on the usual plane-wave Lorenz-Mie theory. ${ }^{11}$ A background in GLMT and its applications may be gained from Refs. 12- 15.

In this formalism, shaped beams such as laser beams are mathematically expanded in terms of partial waves. The complex number that describes the amplitude and the phase of each partial wave in the expansion is called a beam-shape coefficient (BSC). These coefficients may be expressed as angular integrals of the radial component of the beam's electric and magnetic fields. ${ }^{13}$ Unfortunately, none of the

G. Gouesbet and G. Gréhan are with Laboratoire d'Energétique des Systèmes et Procédés, Unité de Recherche Associée Centre National de la Recherche Scientifique No. 230, Complex de Recherche Interdisciplinaire en Aerothermochemie, Institut National des Sciences Appliquées de Rouen, B.P. 08, 76131 M ont-Saint-Aignan, France. J . A. Lock is with the Department of Physics, Cleveland State University, Cleveland, Ohio 44115.

Received 27 J une 1994.

0003-6935/95/122133-11\$06.00/0.

(c) 1995 Optical Society of America. commonly used mathematical descriptions of laser beams is an exact solution of Maxwell's equations. ${ }^{16-18}$ As a result, the BSC's produced by the angular integration of these fiel ds retain a weak dependence on the radial coordinate, ${ }^{19}$ in contrast with the fact that the derivation of the partial-wave decomposition demands that these coefficients be constants. The residual radial dependence is an artifact that results from the imperfect description of the electromagnetic fields of the beam.

On the other hand, a surprisingly accurate approximation to the BSC's is the so-called localized approximation. ${ }^{20}$ It is a simple analytical expression whose accuracy is typically approximately 1 part in $10^{5}$ from the value of the constant portion of the BSC's obtained by numerical integration. ${ }^{21}$ This approximation relies on the localized interpretation of partial-wave expansions, ${ }^{22}$ which is an analogy to van de Hulst's localization principle in Lorenz-Mie theory. ${ }^{23}$ The localization approximation BSC's are constants as is required of partial-wave expansions, and the beam descriptions generated by insertion of these BSC's into the partial-wave expansion have been termed localized beams. ${ }^{19}$ Such beams exactly satisfy Maxwell's equations because they are built from basis functions with constant coefficients.

Until recently, there had been no rigorous justification of the validity of the localized approximation. Initially, its validity was demonstrated by a comparison of the numerical values of the BSC's evaluated by the localized approximation with the values obtained by numerical integration or the finite series method. ${ }^{21,24-26}$ When a beam propagates along the $z$ axis of the coordinate system used to describe the 
partial-wave expansion, i.e., an on-axis beam, one of us recently succeeded in giving a derivation of the localized approximation ${ }^{21}$ that relied on the stationary phase method, in analogy with van de Hulst's derivation of the localization principle. ${ }^{23}$ Thederivation, however, could not be generalized to off-axis beams, i.e., beams that are propagating parallel to but not along the $z$ axis. By the use of another technique that relies on Taylor series expansions, a final derivation of the validity of the localized approximation for a focused Gaussian beam has been re cently obtained for both the on-axis ${ }^{19}$ and the off-axis cases. ${ }^{27}$ This derivation uncovered a modification of the localized approximation that has been called the modified localized approximation.

A significant ingredient in the derivation was the discovery of the so-called standard-beam expressions. Westrongly believe that standard beams will prove to be the best mathematical description of Gaussian beams. Standard beams exactly satisfy Maxwell's equations, because they are constructed from BSC's that are also constants. For the on-axis case, standard-beam BSC's are given by a simple infinite series $^{19}$ that results from an extrapolation of the Davis procedure $^{16}$ for description of the electromagnetic fields of a focused Gaussian beam. The standardbeam BSC's for the off-axis case have not yet been discovered, to our knowledge.

In this paper we consider two aspects of the partialwave representation of laser beams for use in GLMT scattering calculations. (a) In the context of an onaxis focused Gaussian beam, we examine the convergence properties of the infinite series that describes the standard-beam BSC's. We also compare the values of the localized approximation and the modified localized approximation analytical expressions for the BSC's with the standard-beam BSC's that we use as a benchmark. We claim that the closer the localized approximation BSC's come to the standard-beam BSC's, the more accurate the localized approximation is in describing a focused Gaussian beam. (b) We apply the localized interpretation of partial-wave expansions to a top-hat beam and assess the accuracy of the resulting top-hat-beam local ized approximation. To avoid burying the essence of the physics in complicated mathematical expressions, only the on-axis case is considered in this paper.

This paper is organized as follows. Section 2 summarizes the Davis formulation for description of the Gaussian beam electromagnetic fields and introduces the localized and the standard beams. Section 3 compares the numerical values of localized and standard-beam BSC's, providing new insights as to the nature of Gaussian beams and leading to the conclusion that standard beams should indeed be taken as the very definition of Gaussian beams. Section 4 further supports the validity of the localized interpretation of partial-wave analyses by describing the building of localized beams that provide a very satisfactory description of top-hat beams.

\section{Davis, Standard, and Localized Descriptions of} On-Axis Focused Gaussian Beams

\section{A. Davis Formulation}

A description of the electromagnetic fields of a focused Gaussian laser beam is provided by the Davis formulation. 16,17,28 We consider a Gaussian beam that is propagating along the $z^{\prime}$ axis from negative $z^{\prime}$ to positive $z^{\prime}$ (Fig. 1). Two parallel Cartesian coordinate systems must be used in this problem: (a) $\mathbf{X}^{\prime}$, $y^{\prime}, z^{\prime}$, which is attached to the Gaussian beam and whose origin is at the center of the beam waist, and (b) $x, y, z$, which is used to describe the partial-wave expansion of the Gaussian beam. The origin of the $x^{\prime} y^{\prime} z^{\prime}$ system with respect to the $x y z$ system is $z_{0}$. We start by considering the simplest case, $z_{0}=0$, and below we examine the more general on-axis case with $\mathrm{z}_{0} \neq 0$ when appropriate.

We consider a monochromatic light wave with an $\exp (+\mathrm{i} \omega \mathrm{t})$ time dependence. This time dependence will be omitted hereafter, as is the normal practice. In the Davis formulation a laser beam is described by a linearly polarized vector potential,

$$
\mathbf{A}=\left(\mathrm{A}_{\mathrm{x}}, 0,0\right) .
$$

The nonzero component $A_{x}$ is given by

$$
A_{x}=\psi(x, y, z) \exp (-i k z) .
$$

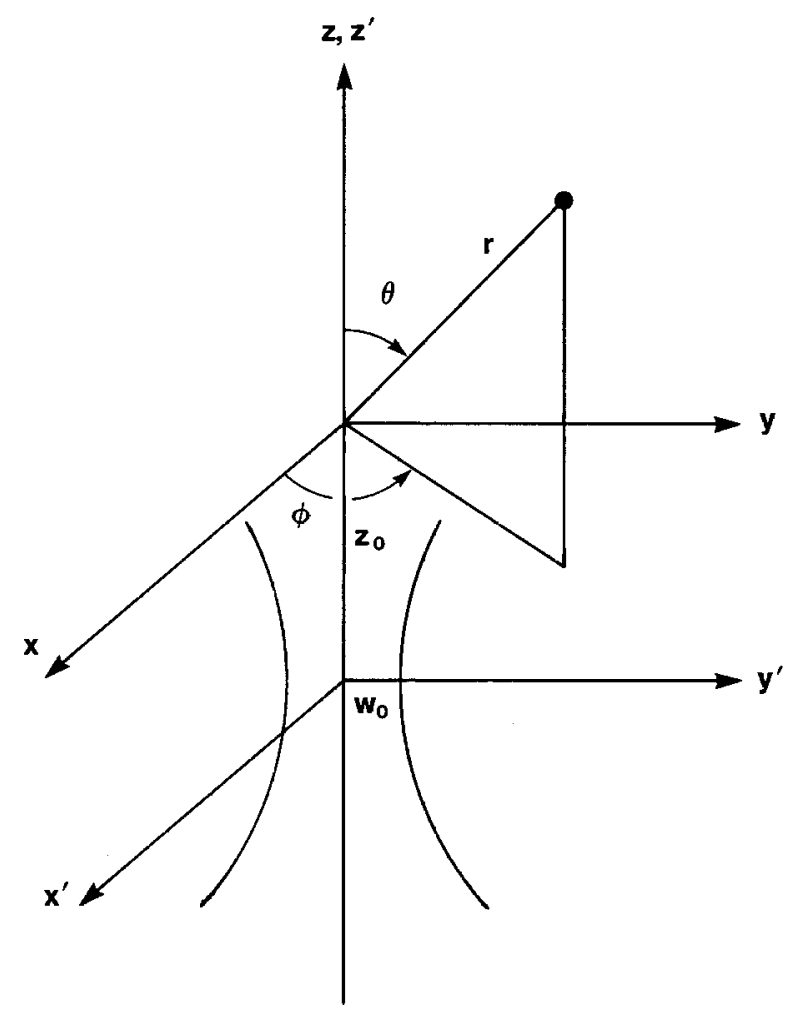

Fig. 1. Two coordinate systems that describe a focused Gaussian beam that is propagating al ong the $z$ axis. The origin of the $x^{\prime} y^{\prime} z^{\prime}$ coordinate system is at the center of the beam waist, and the partial-wave expansion is carried out with respect to the $x, y, z$ coordinate system. 
The function $\psi(x, y, z)$ is unknown and must be determined. Such a determination will involve spatial derivatives. However, the transverse coordinates $x$ and y scale with a small transverse characteristic length $w_{0}$, and the coordinate $z$ scales with a large longitudinal characteristic length I. The scaling lengths $\mathrm{w}_{0}$ and I are taken to be the beam-waist radius and the spreading (or diffraction) length $\mathrm{kw}_{0}{ }^{2}$, respectively. Rescaled dimensionless coordinates $(\xi, \eta, \zeta)$ may therefore be introduced according to

$$
\xi=\frac{x}{w_{0}}, \quad \eta=\frac{y}{w_{0}}, \quad \zeta=\frac{z}{l} .
$$

The rescaled spatial derivatives $\partial \psi / \partial \xi, \partial \psi / \partial \eta$, and $\partial \psi / \partial \zeta$ are now of the same magnitude.

Within the Lorentz gauge, the vector potential A must satisfy the Helmhol tz equation,

$$
\nabla^{2} \mathbf{A}+k^{2} \mathbf{A}=0,
$$

providing the partial differential equation for $\psi$ :

$$
\left(\frac{\partial^{2}}{\partial \xi^{2}}+\frac{\partial^{2}}{\partial \eta^{2}}\right) \psi-2 i \frac{\partial \psi}{\partial \zeta}+s^{2} \frac{\partial^{2} \psi}{\partial \xi^{2}}=0 .
$$

In Eq. (5) we have introduced the small dimensionless parameter s given by

$$
\mathrm{S}=\mathrm{w}_{0} / \mathrm{I}=1 / \mathrm{kw}_{0} .
$$

Because $s$ is the ratio of two characteristic length scales that define the overall aspect of the beam, we name it the beam-confinement factor. For a plane wave with $\mathrm{w}_{0} \rightarrow \infty$, the beam-confinement factor is zero. Even for commonly encountered Gaussian beams, this factor is usually very small. For instance, for $\lambda=0.5 \mu \mathrm{m}$ and $\mathrm{w}_{0}=50 \mu \mathrm{m}$, we have $\mathrm{s} \approx$ $10^{-3}$. There is, however, an upper theoretical limit to $s$ that is discussed at the end of this subsection.

The function $\psi$ is expanded in powers of $s^{2}$ as

$$
\psi=\psi_{0}+s^{2} \psi_{2}+s^{4} \psi_{4}+\ldots
$$

The lowest-order term $\psi_{0}$ represents the fundamental mode of the Gaussian beam. By the use of Eq. (5), it is easily checked that this mode is

$$
\begin{aligned}
& \psi_{0}=\mathrm{iQ} \exp \left[-\mathrm{iQ}\left(\xi^{2}+\eta^{2}\right)\right], \\
& \mathrm{Q}=\frac{1}{\mathrm{i}+2 \zeta} .
\end{aligned}
$$

Once $\psi_{0}$ is known, Eq. (5) implies that the higher-order functions $\psi_{2 n}$ for $n \geq 1$, i.e., corrections to the fundamental mode, may be recursively deduced from

$$
\left(\frac{\partial^{2}}{\partial \xi^{2}}+\frac{\partial^{2}}{\partial \eta^{2}}-2 i \frac{\partial}{\partial \zeta}\right) \psi_{2 n+2}=-\frac{\partial^{2}}{\partial \zeta^{2}} \psi_{2 n}, \quad n \geq 0 .
$$

The functions $\psi_{2}$ and $\psi_{4}$ are more complicated ${ }^{16,17}$ than $\psi_{0}$. Because $\psi_{2}, \psi_{4}, \ldots$ depend on $\psi_{0}$ through
Eq. (10), the fundamental mode $\psi_{0}$ al one completely determines the vector potential A from which electric and magnetic fields are derived by the use of

$$
\begin{aligned}
& \mathbf{E}=\frac{-i c}{k} \nabla(\nabla \cdot \mathbf{A})-i \omega \mathbf{A}, \\
& \mathbf{H}=(\boldsymbol{\nabla} \times \mathbf{A}) / \mu,
\end{aligned}
$$

leading to

$$
\begin{aligned}
& E_{x}=E_{0}\left[\psi_{0}+s^{2}\left(\psi_{2}+\frac{\partial^{2} \psi_{0}}{\partial \xi^{2}}\right)+\ldots\right] \exp (-i k z) \\
& E_{y}=E_{0}\left[s^{2} \frac{\partial^{2} \psi_{0}}{\partial \xi \partial \eta}+s^{4} \frac{\partial^{2} \psi_{2}}{\partial \xi \partial \eta}+\ldots\right] \exp (-i k z) \\
& E_{z}=E_{0}\left[-i s \frac{\partial \psi_{0}}{\partial \xi}-i s^{3}\left(\frac{\partial \psi_{2}}{\partial \xi}+i \frac{\partial^{2} \psi_{0}}{\partial \xi \partial \zeta}\right)+\ldots\right] \exp (-i k z)
\end{aligned}
$$

$\mathrm{H}_{\mathrm{x}}=0$,

$H_{y}=H_{0}\left[\psi_{0}+s^{2}\left(\psi_{2}+i \frac{\partial \psi_{0}}{\partial \zeta}\right)+\ldots\right] \exp (-i k z)$,

$H_{z}=H_{0}\left[-i s \frac{\partial \psi_{0}}{\partial \eta}-i s^{3} \frac{\partial \psi_{2}}{\partial \eta}+\ldots\right] \exp (-i k z)$.

Assume that a Gaussian laser beam is focused to a radius $w_{0}$ equal to $\lambda$ or even $\lambda / 2$, corresponding to $s=$ 0.16 and $s=0.32$, respectively. The so-called diffraction or confinement limit dictates that the beam cannot be focused any more tightly than this. The existence of this limit may be understood in a qualitative and intuitive manner as follows. First consider a plane wave that is propagating in the positive $z$ direction with its electric field polarized in the $x$ direction. The variation of $E_{x}$ in the $z$ direction (i.e., one cycle of variation over the distance $\Delta z=\lambda$ ) induces a magnetic field $\mathrm{H}_{\mathrm{y}}$. Similarly, the variation of $H_{y}$ in the $z$ direction induces a new electric field $E_{x}$. Together the two fields $\mathrm{E}_{\mathrm{x}}$ and $\mathrm{H}_{\mathrm{y}}$ recursively induce each other, causing the forward propagation of the plane wave. Now consider a beam with a Gaussian profile in the $x-y$ plane that is again propagating in the positive $z$ direction. The additional variation of $\mathrm{E}_{x}$ in the $y$ direction induces a new magnetic field $\mathrm{H}_{z}$, and the additional variation of $\mathrm{H}_{\mathrm{y}}$ in the $\mathrm{x}$ direction induces a new electric field $E_{z}$. The variations of $E_{z}$ and $\mathrm{H}_{\mathrm{z}}$ induce yet other fields. Together the new fields $\mathrm{E}_{z}$ and $\mathrm{H}_{\mathrm{z}}$ cause the beam to spread transversely as it propagates. When $\mathrm{E}_{\mathrm{x}}$ and $\mathrm{H}_{\mathrm{y}}$ are sl owly varying in the $x-y$ plane (i.e., $w_{0} \gg \lambda$ or $s \ll 1$ ), the induced fields $E_{z}$ and $H_{z}$ are weak and the spreading is slow. But when $E_{x}$ and $H_{y}$ are as rapidly varying in the $x-y$ plane as they are in the $z$ direction (i.e., $w_{0} \approx \lambda$ or $\mathrm{s} \approx 1 / 2 \pi)$, the induced fields $\mathrm{E}_{z}$ and $\mathrm{H}_{\mathrm{z}}$ are strong, and the transverse spreading of the beam is as rapid as its forward propagation. The transverse spread- 
ing resembles the nearly isotropic radiation from a point source more than it does a transversely localized beam that is propagating in a definite and unambiguous direction. Therefore the range $0.16 \leq \mathrm{s}$ $\leqslant 0.32$ indicates a range for the theoretical limit between the directional propagation of a beam and an isotropically radiating source. A similar limit occurs for Fraunhofer diffraction by an aperture of halfwidth $a$. For $2 a>\lambda$, the diffraction pattern contains both relative maxima and relative minima, indicating direction dependence. But for $2 \mathrm{a} \ll \lambda$, the diffraction pattern is nearly isotropic in the forward hemisphere.

We may also introduce the kth Davis beam approximation defined when only those terms in E qs. (13)-(18) that explicitly depend on $\mathrm{s}$ up to and including the power $\mathrm{s}^{\mathrm{k}}$ are retained. We obtain the first Davis beam $(k=1)$ depending on $\psi_{0}$ and containing terms up to $s^{1}$; the third Davis beam $(k=3)$ depending on $\psi_{0}, \psi_{2}$, and containing terms up to $\mathrm{s}^{3}$; the fifth Davis beam $(\mathrm{k}=5)$ depending on $\psi_{0}, \psi_{2}, \psi_{4}$ and containing terms up to $s^{5}$; and so on. ${ }^{19} \quad$ None of these beams is an exact solution of Maxwell's equations. Maxwell's equations are only satisfied in the limit $k \rightarrow \infty$.

\section{B. Standard Beams}

In the framework of GLMT, an on-axis laser beam is described by the set of BSC's $g_{n}$ given by ${ }^{13}$

$$
\begin{aligned}
g_{n}= & -\frac{1}{2} i^{n-1} \frac{R}{j_{n}(R)} \frac{1}{n(n+1)} \int_{0}^{\pi} \sin ^{2} \theta d \theta f(R, \theta) \\
& \times \exp (-i R \cos \theta) P_{n}{ }^{1}(\cos \theta),
\end{aligned}
$$

in which $r, \theta, \phi$ are spherical coordinates (Fig. 1$), R=$ $\mathrm{kr}, \mathrm{j}_{\mathrm{n}}(\mathrm{R})$ are spherical Bessel functions, $\mathrm{P}_{\mathrm{n}}{ }^{1}$ are associated Legendre polynomials, and $f(R, \theta)$ is defined by

$$
\left(\begin{array}{l}
E_{r} / E_{0} \\
H_{r} / H_{0}
\end{array}\right)=\exp (-i R \cos \theta) f(R, \theta) \sin \theta\left(\begin{array}{c}
\cos \phi \\
\sin \phi
\end{array}\right) .
$$

Consider the kth Davis beam approximation and denote its radial electric and magnetic fields by $E_{r}{ }^{k}$ and $\mathrm{H}_{\mathrm{r}}{ }^{\mathrm{k}}$, respectively, with $\mathrm{k}=1,3,5$ for the first, third, and fifth Davis beams, respectively. These lead to the first-order, third-order, and fifth-order approximations $\mathrm{g}_{\mathrm{n}}{ }^{\mathrm{k}}$ to the BSC's in the following way. ${ }^{19}$ The approximation $\mathrm{f}^{\mathrm{k}}$ to $\mathrm{f}$ is Taylor series expanded in powers of the small parameter $\mathrm{s}$, which permits an analytical integration of Eq. (19). When this is done, it is found that nonconstant terms occur; i.e., the result of the integral does not cancel the prefactor $\mathrm{R} / \mathrm{j}_{n}(\mathrm{R})$, contradicting the fact that the BSC's must be constants. The occurrence of such nonconstant terms is due to the fact that the approximations $\mathrm{E}_{\mathrm{r}}{ }^{\mathrm{k}}$ and $\mathrm{H}_{\mathrm{r}}{ }^{\mathrm{k}}$ do not exactly satisfy the Maxwell equations. However, the nonconstant terms appear at increasingly higher powers of $\mathrm{s}$ when $\mathrm{k}$ increases. For $\mathrm{k}=1$, the $\mathrm{O}\left(\mathrm{s}^{0}\right)$ and $\mathrm{O}\left(\mathrm{s}^{2}\right)$ terms are found to be constants, with nonconstant terms occurring at $\mathrm{O}\left(\mathrm{s}^{4}\right)$ and higher. For $\mathrm{k}=3$, nonconstant terms occur at $\mathrm{O}\left(\mathrm{s}^{8}\right)$ and higher, and they occur at $\mathrm{O}\left(\mathrm{s}^{12}\right)$ and higher for $k=5$. Because we understand that the nonconstant terms are artifacts produced by the approximate nature of the beam descriptions, they may be dismissed. Because the details of the computations require much algebra, it is somewhat of a pleasant surprise that the resulting $g_{n}{ }^{{ }^{k}} s$ for $k=1,3,5$ may be written in the simple form,

$$
g_{n}{ }^{k}=\sum_{l=0}^{k}(-1)^{\prime} \frac{s^{2 l}}{I !} \frac{(n-1) !}{(n-1-1) !} \frac{(n+1+1) !}{(n+1) !},
$$

which explicitly leads to

$$
\begin{aligned}
g_{n}{ }^{1}= & 1-(n-1)(n+2) s^{2}, \\
g_{n}{ }^{3}= & g_{n}{ }^{1}+1 / 2(n-2)(n-1)(n+2)(n+3) s^{4} \\
& -1 / 6(n-3)(n-2)(n-1)(n+2)(n+3) \\
& \times(n+4) s^{6}, \\
g_{n}{ }^{5}= & g_{n}{ }^{3}+1 / 24(n-4)(n-3)(n-2)(n-1)(n+2) \\
& \times(n+3)(n+4)(n+5) s^{8}-1 / 120(n-5) \ldots \\
& \times(n-1)(n+2) \ldots(n+6) s^{10} .
\end{aligned}
$$

The same procedure may be carried out for the general on-axis case with $z_{0} \neq 0$. The amount of algebra is, however, now so great that by-hand computations are unreasonable, and use of symbolic computation software such as MAPLE is compulsory. Still, the $g_{n}{ }^{k}$ 's end up being given by the relatively simple expression ${ }^{19}$

$$
\begin{aligned}
g_{n}{ }^{k}= & \sum_{j=0}^{j+2 l=} \sum_{l=0}^{2 k+1}\left(\frac{-2 i s z_{0}}{w_{0}}\right)^{j}(-1)^{I} s^{2 l} \frac{(I+j) !}{I ! j !} \frac{1}{I !} \\
& \times \frac{(n-1) !}{(n-1-l) !} \frac{(n+1+I) !}{(n+1) !} \exp \left(i k z_{0}\right) .
\end{aligned}
$$

Equation (25) represents the most general and rigorous result for the analytical evaluation of the BSC's, which we call the s-expansion method.

Although $\mathrm{s}$ is usually small, it is demonstrated in Subsection 2.C. that even $g_{n}{ }^{5}$, which contains terms of up to $\mathrm{O}\left(\mathrm{s}^{10}\right)$, is not sufficient to describe extremely focused beams or the BSC's accurately for large partial waves. Because the amount of algebra that would be required for higher-order Davis beams to be designed and the corresponding $g_{n}{ }^{k}$ 's to be evaluated is extensive, it is appealing to conjecture that Eqs. (21) and (25) remain valid for $k>5$. The infinite generalization then reads

$$
\begin{aligned}
g_{n}^{\infty}= & \sum_{j=0}^{\infty} \sum_{l=0}^{\infty}\left(-2 i s \frac{z_{0}}{w_{0}}\right)^{j}(-1)^{\prime} s^{2 l} \frac{(I+j) !}{I ! j !} \frac{1}{I !} \\
& \times \frac{(n-1) !}{(n-1-I) !} \frac{(n+1+I) !}{(n+1) !} \exp \left(i k z_{0}\right),
\end{aligned}
$$


which reduces to

$$
g_{n}^{\infty}=\sum_{l=0}^{\infty} \frac{(-1)^{1} s^{2 l}}{I !} \frac{(n-1) !}{(n-1-I) !} \frac{(n+1+I) !}{(n+1) !}
$$

for $z_{0}=0$.

We call Eqs. (26) and (27) the standard BSC's, and the beam defined by this set of BSC's is called a standard beam. The $g_{n}{ }^{k}$ coefficients of E qs. (21) and (25) will be called the kth-order approximation to the standard BSC's. We claim that standard beams should be taken as the ideal description of Gaussian beams. This claim will be reinforced by the numerical results of Section 3. But before proceeding to these numerical results, we must introduce the localized approximation to the BSC's of Eq. (19).

\section{Localized Approximation}

The localized approximation for a focused Gaussian beam ${ }^{20}$ is built on the first-order Davis beam of Eqs. (8) and $(9)$ and results from the localized interpretation of partial-wave expansions. For $z_{0}=0$, the radial electric field of the first-order Davis beam in Eq. (11) may be written as

$$
E_{r}=E_{0} \exp (-i k z) \sin \theta \cos \phi f(k r, \theta),
$$

with

$$
f(k r, \theta)=i Q \exp \left(-i Q \frac{r^{2} \sin ^{2} \theta}{w_{0}^{2}}\right)\left(1-2 Q s r \cos \theta / w_{0}\right) \text {. }
$$

The localized approximation $\bar{g}_{n}$ to the BSC's $g_{n}$ is obtained by application of the localization operator $L$ to the function $\mathrm{f}$ in $\mathrm{Eq}$. (29) according to the prescription

$$
\hat{L} f(R, \theta)=f(n+1 / 2, \pi / 2),
$$

which is the van de Hulst localization principle applied in the focal plane of the beam. ${ }^{22}$ The integration in Eq. (19) may then be easily performed, ${ }^{21}$ yielding

$$
\overline{g_{n}}=\exp \left[-s^{2}(n+1 / 2)^{2}\right] .
$$

To motivate the modified localized approximation, we now demonstrate that the standard BSC's $g_{n}{ }^{\infty}$ of Eq. (27) may be approximated by

$$
g_{n}^{\infty} \approx \exp \left[-s^{2}(n-1)(n+2)\right] .
$$

The demonstration proceeds in the following way. The exponential in Eq. (32) may be expanded as

$$
\begin{aligned}
\exp & \left.-s^{2}(n-1)(n+2)\right] \\
= & g_{n}{ }^{1}+1 / 2 \alpha(n-2)(n-1)(n+2)(n+3) s^{4} \\
& -1 / 6 \beta(n-3)(n-2)(n-1)(n+2)(n+3) \\
& \times(n+4) s^{6}+\ldots,
\end{aligned}
$$

where $\mathrm{g}_{\mathrm{n}}{ }^{1}$ is given by Eq. (22) and

$$
\begin{aligned}
& \alpha=\frac{(n-1)(n+2)}{(n-2)(n+3)}, \\
& \beta=\frac{(n-1)^{2}(n+2)^{2}}{(n-3)(n-2)(n+3)(n+4)} .
\end{aligned}
$$

The behavior of $\alpha$ and $\beta$ as a function of the partial wave $\mathrm{n}$ is illustrated in Table 1 . For small partial waves, when $\alpha$ and $\beta$ are significantly different from 1 , the difference between the exponential in relation (32) expanded up to $\mathrm{O}\left(s^{6}\right)$ and $\mathrm{g}_{\mathrm{n}}{ }^{\infty}$ is small because $\mathrm{s}^{4}$ and $s^{6}$ are small $\left(10^{-12}\right.$ and $10^{-18}$ for a typical beam with $\left.\mathrm{s}=10^{-3}\right)$. For large partial waves, the $\mathrm{O}\left(\mathrm{s}^{4}\right)$ and $\mathrm{O}\left(\mathrm{s}^{6}\right)$ terms contribute significantly. But then $\alpha \approx \beta$ $\approx 1$ with a high accuracy, again validating relation (32). The same argument holds for higher powers of $s$ as well.

Relation (32) implies that we may introduce a modified local ized approxi mation and a modi fied localization operator,

$$
\hat{L} \bmod f(R, \theta)=f\left[(n-1)^{1 / 2}(n+2)^{1 / 2}, \pi / 2\right],
$$

leading to

$$
\overline{g_{n, \bmod }}=\exp \left[-s^{2}(n-1)(n+2)\right] .
$$

The modified localized approximation may also be written as

$$
\overline{g_{n, \bmod }}=\exp \left[-s^{2} \gamma(n+1 / 2)^{2}\right],
$$

where

$$
\gamma=\frac{(n-1)(n+2)}{(n+1 / 2)^{2}}
$$

As is shown in Table 1, this ratio al so quickly tends to 1 as $n$ increases. Ther efore the local ized approximation of Eq. (31) is very close to the modified localized approximation of Eq. (37). For $z_{0} \neq 0$, these approxi-

Table 1. Coefficients $\alpha, \beta$, and $\gamma$ of Eqs. (34), (35), and (39), Respectively, as a Function of Partial Wave

\begin{tabular}{cccc}
\hline Partial Wave & $\alpha[$ Eq. (34)] & $\beta[$ Eq. (35)] & $\gamma[$ Eq. (39)] \\
\hline 5 & 1.166667 & 1.814815 & 0.925620 \\
10 & 1.038462 & 1.144427 & 0.979592 \\
50 & 1.001573 & 1.005518 & 0.999118 \\
100 & 1.000396 & 1.001388 & 0.999777 \\
500 & 1.000016 & 1.000056 & 0.999991 \\
1000 & 1.000004 & 1.000014 & 0.999998 \\
\hline
\end{tabular}

20 April 1995 / Vol. 34, No. 12 / APPLIED OPTICS 
mations generalize to

$$
\begin{aligned}
\overline{g_{n}}= & \left(1+2 i s \frac{z_{0}}{w_{0}}\right)^{-1} \exp \left(i k z_{0}\right) \\
& \times \exp \left[\frac{-s^{2}(n+1 / 2)^{2}}{1+2 i s z_{0} / w_{0}}\right], \\
\overline{g_{n, \text { mod }}}= & \left(1+2 i s \frac{z_{0}}{w_{0}}\right)^{-1} \exp \left(i k z_{0}\right) \\
& \times \exp \left[\frac{-s^{2}(n-1)(n+2)}{1+2 i s z_{0} / w_{0}}\right] .
\end{aligned}
$$

The results shown in Table 1 indicate that the analytical expressions of the localized and the modified localized approximations bear a strong resemblance to the infinite-series standard-beam BSC's. But Eqs. (31) and (37) are built on the first-order beam, and the standard beams incorporateall thehigher-order terms. It is a pleasant surprise that the localized approximation that is built on the first-order beam anticipates these higher-order descriptions and includes them in an approximate way. This will prove to be very useful when the standard-beam BSC's are slowly convergent and is examined in more detail in the Section 3.

\section{Numerical Discussion of Localized and Standard BSC's}

If we insert the localized and the standard BSC's into the beam partial-wave expansions, we generate the localized and the standard beams, respectively. We could then compare the localized- and the standardbeam profiles. This was done for the beam focal waist plane in Refs. 19 and 27. In this paper, instead, we emphasize the comparison between the individual values of the localized and the standard BSC's.

\section{A. Comparison for $z_{0}=0$ and $s \ll 1$}

For the case $z_{0}=0$, we compare the values of the BSC's in Tables 2 and 3 for (1) the localized approximation of Eq. (31), labeled LA; (2) the modified localized approximation of Eq. (37) labeled MLA; and
(3) the s-expansion method for the first-, third-, and fifth-order approximations to the standard BSC's of Eqs. (22-24). These are labeled D1, D3, D5, respectively. Also compared are (4) the standard-beam values obtained from Eq. (21) when $\mathrm{k}$ is increased until a convergence of 9 significant figures is achieved. The values of $k$ for convergence is listed in Tables 2 and 3 , as is the numerical value of $g_{n}{ }^{\infty}$. The results shown in Table 2 are for the commonly encountered situation of $\mathrm{s}=0.001$.

First we consider the convergence of Eq. (21) for the standard-beam BSC's for $\mathrm{S}=0.001$, which are typical of laser Doppler and phase Doppler instruments, ${ }^{1-3}$ and that correspond to focusing of the beam to a radius $w_{0} \approx 150 \lambda$. The evaluation of $g_{n}{ }^{k}$ has been carried out by the use of the symbolic computation software MAPLE. This is compulsory because when one is evaluating the standard coefficients, the number of digits required in the computations to obtain 9 significant figures in the results may be far beyond what is available with FORTRAN double precision variables. MAPLE allows one to carry out evaluations with an arbitrary number of significant figures, which is only limited by the host-computer available storage, by setting the MAPLE variable digits to a prescribed value. For instance, digits $=12$ is enough to evaluate $g_{n}{ }^{k}$ for small $n$. For $n=2500$, the evaluation of $g_{n}{ }^{31}$ requires digits $=20$, and for $n=5000$, the evaluation of $\mathrm{g}_{\mathrm{n}}{ }^{101}$ requires digits $=40$. Therefore, although the standard BSC's provide benchmark values for the BSC's that describe a focused Gaussian beam, such benchmark values may in practice be difficult to obtain for large partial waves and tightly confined beams.

Examining the sequence of $g_{n}{ }^{k}$ 's for various partial waves $n$ and various beam orders $k$, we may follow the convergence of the standard scheme. Up to the partial wave $n=5, g_{n}{ }^{1}$ is sufficient; i.e., the standard BSC's are correctly evaluated only by the use of first-order Davis beam. For $10 \leqq \mathrm{n} \lesssim 100$, the use of the third-order Davis beam is required. Eventually it is necessary to rely on the kth-order standard scheme with $\mathrm{k}$ values larger than 5 . For example, for $n=1000,2500$, and 5000 we need $k=15,31$, and 101, respectively. Large partial waves $n$ are associ-

Table 2. BSC's as a Function of Partial Wave for $s=0.001$ for the Localized Approximation (LA); the Modified Localized Approximation (MLA); the First(D1), Third- (D3), and Fifth-order (D5) approximations to the Standard Beam; and the Standard Beam (S) ${ }^{a}$

\begin{tabular}{rcccccc}
\hline $\mathrm{n}$ & LA & MLA & D1 & D3 & D5 & k, S \\
\hline 1 & 0.999997750 & 1.000000000 & 1.000000000 & 1.000000000 & 1.000000000 & 1 , Same as D1 \\
2 & 0.999993750 & 0.999996000 & 0.999996000 & 0.999996000 & 0.999996000 & 1 , Same as D1 \\
5 & 0.999969750 & 0.999972000 & 0.999972000 & 0.999972000 & 0.999972000 & 1 , Same as D1 \\
10 & 0.999889756 & 0.999892006 & 0.999892000 & 0.999892006 & 0.999892006 & 3, Same as D3 \\
50 & 0.997452999 & 0.997455243 & 0.999452000 & 0.999455238 & 0.997455238 & 3, Same as D3 \\
100 & 0.989950586 & 0.989952813 & 0.080902000 & 0.989952793 & 0.989952793 & 3, Same as D3 \\
1000 & 0.367511653 & 0.367512480 & $<0$ & 0.332834669 & 0.366292083 & $15,0.367511867$ \\
2500 & 0.001925633 & 0.001925638 & $<0$ & $<0$ & $<0$ & $31,0.001925639$ \\
5000 & $0.138186 \times 10^{-10}$ & $0.138187 \times 10^{-10}$ & $<0$ & $<0$ & $<0$ & $101,0.138208 \times 10^{-10}$ \\
\hline
\end{tabular}

${ }^{a}$ or the standard beam, the number of terms in the infinite series of Eq. (27) required for convergence to 9 significant figures ( $\mathrm{k}$ ) is also given. 
Table 3. BSC's as a Function of Partial Wave for $s=0.16$ for the Localized Approximation (LA); the Modified Localized Approximation (MLA); the First- (D1), Third- (D3), and Fifth-Order (D5) Approximations to the Standard Beam; and the Standard Beam (S) ${ }^{a}$

\begin{tabular}{rcccccc}
\hline $\mathrm{n}$ & $\mathrm{LA}$ & $\mathrm{MLA}$ & $\mathrm{D} 1$ & $\mathrm{D} 3$ & $\mathrm{D} 5$ & $\mathrm{k}, \mathrm{S}$ \\
\hline 1 & 0.944027482 & 1.000000000 & 1.000000000 & 1.000000000 & 1.000000000 & 1, Same as D1 \\
2 & 0.852143789 & 0.902668412 & 0.897600000 & 0.897600000 & 0.897600000 & 1 , Same as D1 \\
4 & 0.595472542 & 0.630778820 & 0.539200000 & 0.616138215 & 0.618138215 & 3 , Same as D3 \\
6 & 0.339052607 & 0.359155441 & $<0$ & 0.327063245 & 0.343026339 & 5 , Same as D5 \\
10 & 0.059463060 & 0.062988600 & $<0$ & $<0$ & $<0$ & $9,0.058365667$ \\
15 & 0.002132629 & 0.002259075 & $<0$ & $<0$ & $<0$ & $15,0.002267813$ \\
20 & 0.000021266 & 0.000022526 & $<0$ & $<0$ & $<0$ & $19,0.000031912$ \\
25 & $0.589603 \times 10^{-7}$ & $0.624562 \times 10^{-7}$ & $<0$ & $<0$ & $<0$ & $25,1.853835 \times 10^{-7}$
\end{tabular}

aF or the standard beam, the number of terms in the infinite series of Eq. (27) required for convergence to 9 significant figures (k) is also given.

ated through the local ized interpretation with geometric light rays that are passing far from the beam axis. ${ }^{22,23}$ Therefore the description of the outer parts of the beam requires higher k orders than the description of the central region. This observation must be reconciled with the previously demonstrated fact ${ }^{18}$ that a first-order Davis beam satisfies Maxwell's equations up to $\mathrm{O}\left(\mathrm{s}^{2}\right)$ uniformly over all space. Our results on the convergence of the standard scheme in Table 2 indicate that the situation is more subtle because the coefficients $\alpha_{k}$ of the various powers of $s$ can make terms such as $\alpha_{k} s^{k}, k>2$, significant if $\alpha_{k}$ is an increasing function of the partial wave $n$ and if $n$ is big enough. Clearly, for the on-axis case, geometric rays associated with large partial waves possess vanishingly small amplitudes that are ineffective in the light-scattering process, so that a poor evaluation of the corresponding BSC's should not be influential. Note, however, that whether a partial wave is effective also depends on the size of the target particle through the Lorenz-Mie partial-wave scattering amplitudes $a_{n}$ and $b_{n}$. In addition, low partial waves are classically associated with backscattering, and large partial waves with sidescattering. Thus, when one compares $\mathrm{g}_{\mathrm{n}}$ values such as those in Table 2, where $g_{1} \approx 1.0$ and $g_{5000} \approx 10^{-11}$, which is vanishingly small in comparison with $g_{1}$, we should actually compare the light scattered in different directions. A more refined discussion should then take into consideration scattering diagrams in an actual scattering process.

We now consider the accuracy of the localized approximation and the modified local ization approximation BSC's when compared against the benchmark standard-beam BSC's for $s=0.001$. In Table 2, the comparison between the modified localized approximation and the standard scheme is excellent. Up to the partial wave $n=100$, the difference between the modified localized approximation and the standard scheme typically does not exceed 1 part in $10^{8}$. Even for $n=5000$, the disagreement lies in the fifth significant figure. There the modified localized approximation based on the first-order Davis beam anticipates the information contained in the 101st order of the standard scheme. This unexpected internal coherence is considered as a cross-check of the validity of the modified localized approximation, and of the fact that standard beams should be considered as the ideal reference beams. Finally, the localized approximation agrees reasonably well with the standard BSC values. But the agreement for the modified localized approximation is better, especially for $\mathrm{n}<100$.

\section{B. Comparison for $z_{0}=0$ and $s=0.16$}

Table 3 now provides a comparison for $z_{0}=0$ and $\mathrm{s}=$ 0.16 [i.e., $1 /(2 \pi)$ ] near the theoretical confinement limit. The range of important partial waves is much smaller than in Table 2. This is a direct consequence of the localized interpretation; i.e., a BSC of partial wave $n$ is associated with the amplitude of the geometric light ray that is passing at a distance

$$
\rho_{\mathrm{n}}=\frac{(\mathrm{n}+1 / 2) \lambda}{2 \pi}
$$

from the beam axis at the focal waist. From relation (32) it can be seen that the amplitude decreases to $1 / \mathrm{e}^{2}$ of its value on the $z$ axis for $n \approx 1500$ if $s=10^{-3}$ and for $\mathrm{n} \approx 10$ if $\mathrm{s}=0.16$. The $\mathrm{n}$ values are strongly correlated with the necessity of using bigger k orders to obtain convergence for the standard-beam BSC's.

There is also an increase in the difference between the modified localized approximation and the standard values. This difference is dramatic for $n=25$. There is also an increased difference between the localized and the modified localized approximations. Also, depending on the partial wave $n$, the modified localized approximation may compare more favorably or less favorably with the standard BSC values than the localized approximation. The deterioration of the comparisons for $\mathrm{s}=0.16$ is consistent with the approach to the physical confinement limit.

\section{Comparison for $z_{0} \neq 0$}

We now focus our attention on the convergence of the standard-beam BSC's for the general on-axis case $\mathrm{z}_{0} \neq 0$, with $\mathrm{g}_{\mathrm{n}}{ }^{\infty}$ given by Eq. (26). Computations are carried out by means of a MAPLE procedure, increasing $\mathrm{k}$ in Eq. (25) until the convergence test $g_{n}{ }^{k}=g_{n}{ }^{k+2}$ is satisfied to an accuracy of 50 significant figures. 
The value of $k$ for which convergence is reached is denoted by $\mathrm{K}$. In Fig. 2, $\mathrm{K}$ is displayed versus $\mathrm{Z}_{0}$ for $g_{1}^{\infty}$, the BSC for the first partial wave. The beamwaist radius $\mathrm{w}_{0}$ is used as a parameter. The number of terms required for convergence increases when $z_{0}$ increases and when $w_{0}$ decreases, i.e., when the focal waist of a tightly focused beam is far upstream or downstream from the origin of coordinates. The increase versus $z_{0}$ is particularly sharp for the most focused beam $\left(w_{0}=0.25 \mu \mathrm{m}, \mathrm{s}=0.32\right)$ at the upper limit of the physical confinement range. Figure 3 presents the same data shown in Fig. 2 versus the dimensionless quantity $z_{0} / I$, in which $I$ is the spreading length. Because of the fact that $I$ is the natural characteristic length to rescale the $z$ coordinate, all the curves in Fig. 2 collapse to a single curve in Fig. 3.

In Figs. 2 and 3 only the first partial wave was considered. To extend the analysis to all partial waves, $\mathrm{K}$ is presented in Fig. 4 as $\mathrm{K}\left(\mathrm{D}, \mathrm{z}_{0} / \mathrm{I}\right)$. In Fig. 4 $z_{0}$ is still rescaled by l. Rather than the partial wave $\mathrm{n}$, the ordinate is now taken to be

$$
D=(n-1)^{1 / 2}(n+2)^{1 / 2} S,
$$

which is the distance $\rho$ from the beam axis associated with $\mathrm{n}$ through the modified localized interpretation [Eq. (44), below] and rescaled by $w_{0}$. Figure 4, for $\lambda=0.5 \mu \mathrm{m}, \mathrm{w}_{0}=5 \mu \mathrm{m}$, and $\mathrm{s}=0.016$, demonstrates how the number of terms required for convergence increases when $z_{0}$ or $n$ increases.

To some extent, these results might be considered troubling. Consider, for instance, a small value for $\mathrm{s}$, which leads us to expect that the beam may be safely described by a first-order Davis beam. Figure 4, however, tells us that this conclusion is true only in a small region that surrounds the beam-waist center and that the standard-beam BSC's will be slowly convergent otherwise. Fortunately, as mentioned above, the localized approximation anticipates the behavior of high-order Davis beams and therefore may be used in this case as an alternative to the

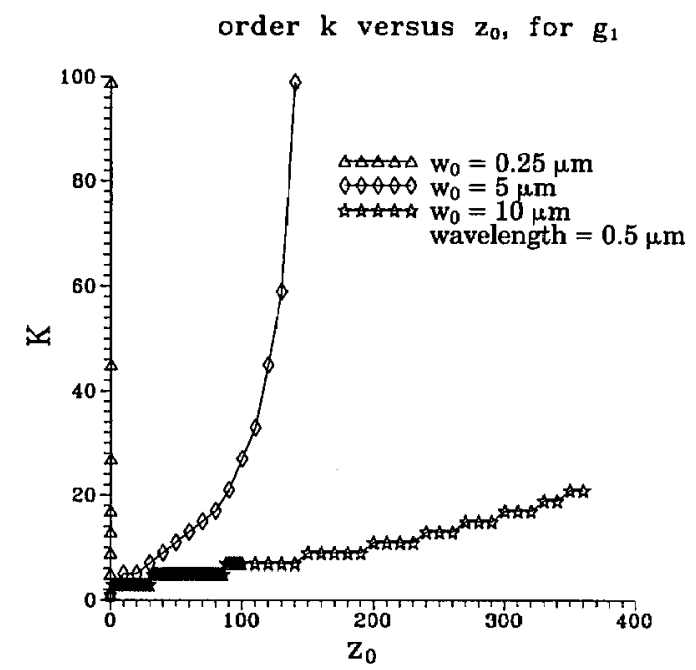

Fig. 2. Value $\mathrm{K}$ of the kth-order standard beam required for convergence of the BSC $g_{1}^{\infty}$ as a function of $\mathrm{z}_{0}$.

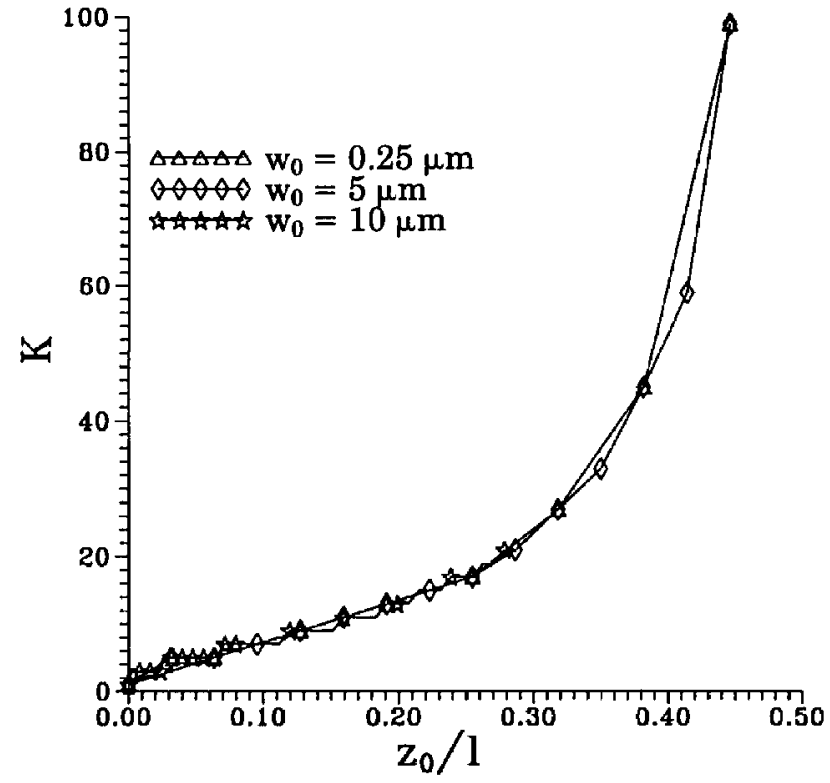

Fig. 3. Value $K$ of the kth-order standard beam required for the convergence of the BSC $g_{1}^{\infty}$ as a function of $z_{0} / \mathrm{l}$. The individual curves from Fig. 2 now coincide.

standard-beam procedure to provide a fast and accurate way to evaluate BSC's. This is the most important result of this paper. When one is computing light scattering with the GLMT formalism, the description of the incident beam should be accurate and should permit scattering calculations to be performed rapidly. The standard-beam BSC's yield the best description of the beam. But in certain circumstances their slow convergence causes a large increase in the computer run time of scattering calculations. The localized approximation, on the other hand, satisfies both criteria of accuracy and computational speed, making it a useful tool in GLMT calculations.

The comparison between the standard, localized, and modified localized BSC's may be complicated in

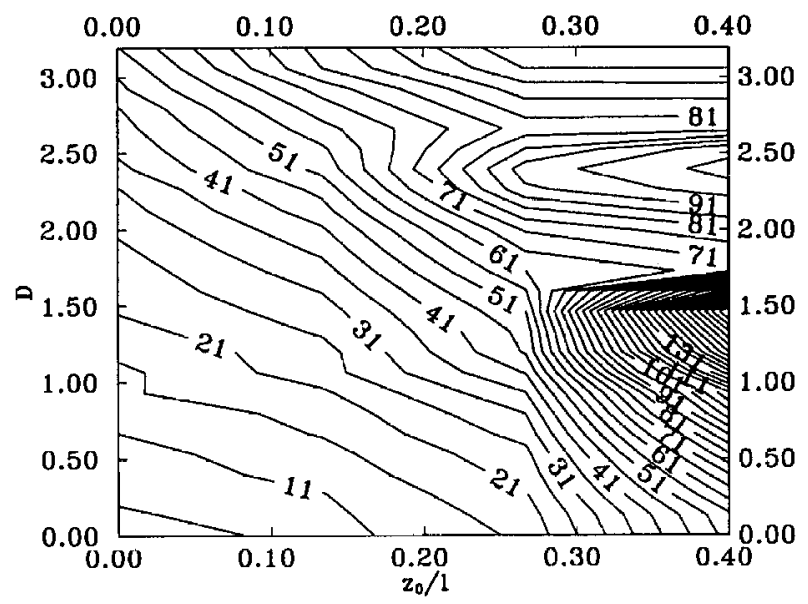

Fig. 4. Value $K$ of the kth-order standard beam required for the convergence of the BSC $g_{n}^{\infty}$ as a function of $z_{0} / I$ and $D=$ $s(n-1)^{1 / 2}(n+2)^{1 / 2}$ for $\lambda=0.5 \mu m, w_{0}=5 \mu m$, and $s=0.016$. 
the so-called off-axis case, when only one side of the particle is illuminated by the beam. To investigate this problem, it is necessary to design an expansion method for higher-order Davis beams and corresponding off-axis standard-beam expressions. As of yet, this task has not been undertaken, to our knowledge.

\section{Top-Hat Beams}

Originally, the localized approximation for a focused Gaussian beam was obtained by analogy to van de Hulst's localization principle for plane waves. This led us to associate a BSC $\mathrm{g}_{n}$ with the amplitude of a geometric light ray that is passing at a distance $\rho_{\mathrm{n}}$ from the beam axis, in which $\rho_{n}$ is given by $\mathrm{Eq}$. (42). In the modified localized approximation, we have

$$
\rho_{\mathrm{n}}=(\mathrm{n}-1)^{1 / 2}(\mathrm{n}+2)^{1 / 2} \frac{\lambda}{2 \pi} .
$$

These relations allow us to investigate light scattering by the use of the localized approximation for beams more general than Gaussian beams. For instance, consider top-hat beams, which are used in certain optical particle-sizing instruments. ${ }^{7-10}$ Tophat beam scattering has been calculated previously with the localized approximation, without, however, directly demonstration of the validity of the localized approach to the case of top-hat beams. ${ }^{10}$ An assessment of this validity is provided here, illustrating the potentialities of thelocalized interpretation. Weconsider an idealized beam profile defined by $\mathrm{Eq}$. (20) in which

$$
f(k r, \theta)= \begin{cases}1 & \text { if } r \sin \theta \leq w_{0} \\ 0 & \text { if } r \sin \theta>w_{0}\end{cases}
$$

corresponding to the plateau region of constant illumination of radius $w_{0}$ around the beam axis. If we rely on the localized interpretation of Eq. (30), such a beam should be generated by localized BSC's given by

$$
\bar{g}_{n}=\left\{\begin{array}{ll}
1 & \text { if } n \leq k w_{0}-1 / 2 \\
0 & \text { if } n>k w_{0}-1 / 2
\end{array} .\right.
$$

The accuracy to which Eq. (46) describes a top-hat beam was tested in the following way. It is known that a given set of BSC's $g_{n}$ defines an on-axis laser beam that is an exact solution of Maxwell's equations. The electric-field components of this beam in the beam-waist plane (i.e., $\theta=\pi / 2$ ) are given by

$$
\begin{aligned}
& E_{x}(k r, \pi / 2, \phi)=F_{1}(k r)-F_{2}(k r) \sin ^{2} \phi, \\
& E_{y}(k r, \pi / 2, \phi)=F_{2}(k r) \sin \phi \cos \phi, \\
& E_{z}(k r, \pi / 2, \phi)=F_{3}(k r) \cos \phi,
\end{aligned}
$$

with similar relations for the magnetic fields' components. The function $\mathrm{F}_{1}$ describes the dominant shape of the beam profile, and $F_{2}$ and $F_{3}$ denote corrections to the dominant shape induced by variations in $\mathbf{E}$ and
$\mathbf{H}$ in the $x-y$ plane. The explicit forms of the functions $F_{1}, F_{2}, F_{3}$ in terms of the BSC's are given in Ref. 19.

For the localized beam model of Eq. (46) the dominant shape function $F_{1}(k r)$ was calculated for the top-hat profile laser beam, for $\mathrm{w}_{0}=25 \mu \mathrm{m}, 7.5 \mu \mathrm{m}$, and $2.5 \mu \mathrm{m}$, and is shown in Figs. 5(a)-5(c). The results are generally encouraging. The fields are virtually constant from the $z$ axis out to the radius $w_{0}$ in the $x-y$ plane as hoped. But beyond $w_{0}$, instead of being rigorously zero, the fields are oscillatory, with a slowly decreasing amplitude. This is reminiscent of the oscillatory ringing in the Fourier transform of a function with a hard edge. ${ }^{29}$ As the radius of the top-hat beam $w_{0}$ decreases, the oscillations become coarser and their amplitude increases. For example, when $\mathrm{w}_{0}=25 \mu \mathrm{m}$, the amplitude of the oscillations falls by an order of magnitude from its value in the plateau region when the distance $\rho$ from the $z$ axis is approximately $1.2 \mathrm{w}_{0}$. For $\mathrm{w}_{0}=2.5 \mu \mathrm{m}$ this occurs when $\rho \approx 1.7 w_{0}$. The exact effect that these oscillations in the local ized electric and magnetic fiel ds have on the far-field scattered intensity calculated in the GLMT framework is not known. But it is expected to be small because the amplitude of the oscillations is only a small fraction of $E_{0}$, the field strength in the plateau region.

The sharp cutoff in Eq. (45), however, is not observed in oscilloscope traces of experimental beam profiles. ${ }^{7}$ In actuality, the fields possess a smooth but rapid roll-off for $\rho>w_{0}$. A more realistic model of a top-hat beam is then

$$
f(k r, \theta)= \begin{cases}1 & \text { if } r \sin \theta \leq w_{0} \\ \exp \left[-\left(r \sin \theta-w_{0}\right)^{2} / \epsilon^{2}\right] & \text { if } r \sin \theta>w_{0},\end{cases}
$$

where $\epsilon$ is the small roll-off distance of the beam in the $x$-y plane. Thelocalized beam model for Eq. (50) with the prescription of Eq. (30) is then

$$
\bar{g}_{n}= \begin{cases}1 & \text { if } n \leq k w_{0}-1 / 2 \\ \exp \left[-\left(n+1 / 2-k w_{0}\right)^{2} / k^{2} \epsilon^{2}\right] & \text { if } n>k w_{0}-1 / 2 .\end{cases}
$$

The dominant shape function $F_{1}$ of the beam defined by Eq. (51) was calculated for $\mathrm{w}_{0}=25 \mu \mathrm{m}$ and $\epsilon=$ $0.05 w_{0}$, and is shown in Fig. $6(\mathrm{a})$. The oscillations in the field persist for $\rho \geq w_{0}$. But their amplitude has now been decreased to less than $10^{-3} \mathrm{E}_{0}$, and their effect on the far-field scattered intensity is similarly reduced. This decrease is confirmed in Fig. 6(b) for $\mathrm{w}_{0}=25 \mu \mathrm{m}$ with the more gradual roll-off $\epsilon=0.1 \mathrm{w}_{0}$. The oscillation level has now decreased to less than $10^{-4} \mathrm{E}_{0}$ and is again reminiscent of the reduction in the oscillatory ringing in the Fourier transform of a function with a smooth, gradual edge. ${ }^{29}$ The results in this section therefore further support the localized interpretation of partial-wave analyses and provide a 


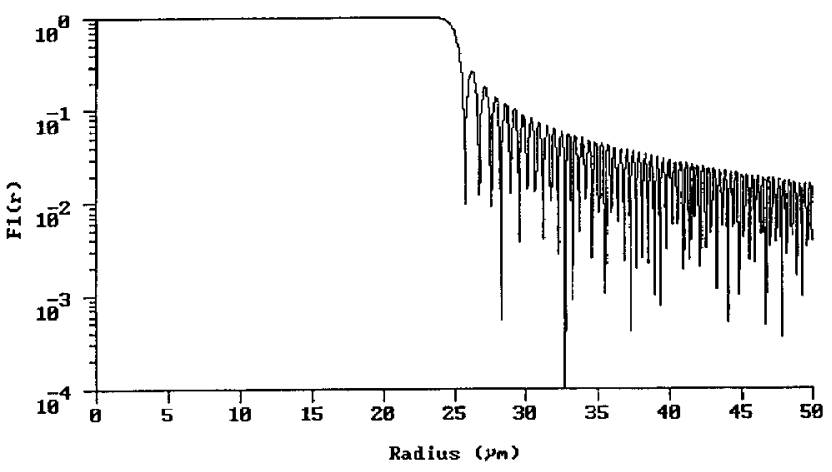

(a)

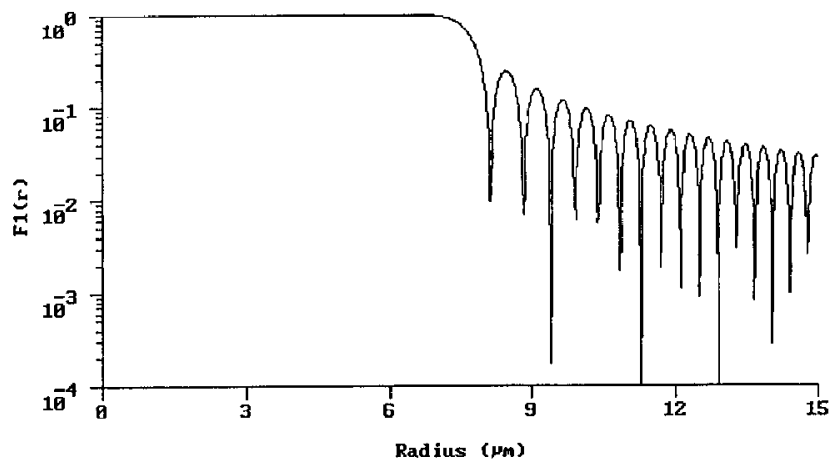

(b)

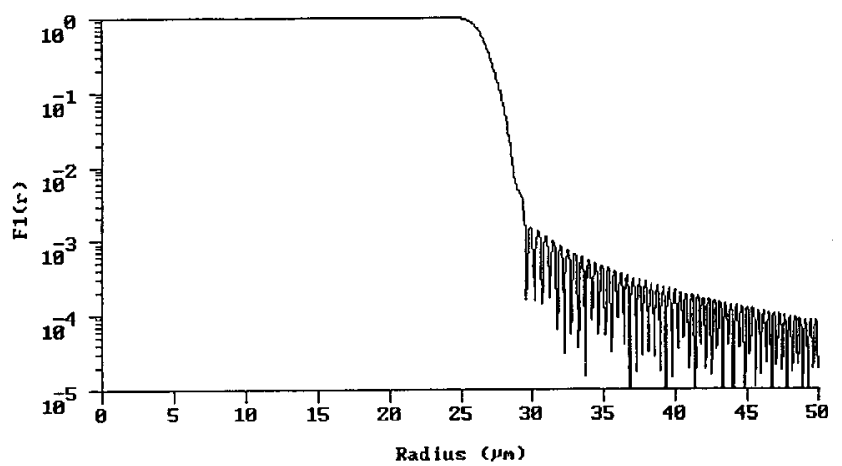

(a)

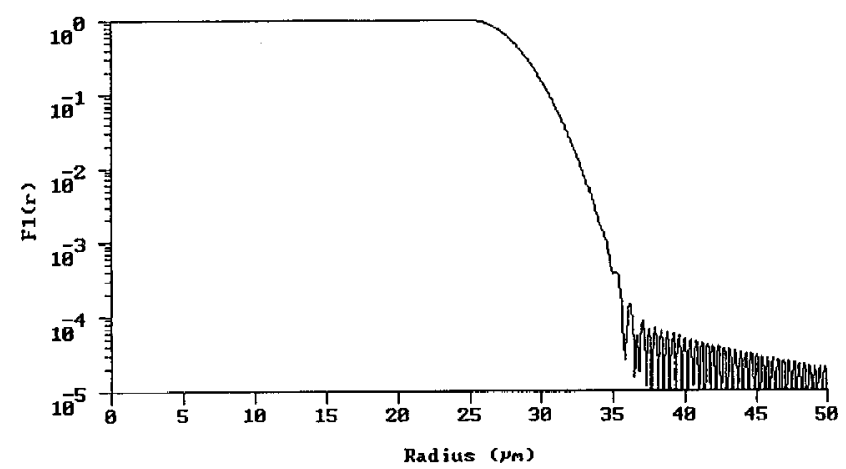

(b)

Fig. 6. Dominant beam-shape function $F_{1}$ for the localized tophat beam of Eq. (50), which possesses a smooth roll-off of width $\epsilon$. The curves are for $w_{0}=25 \mu \mathrm{m}$ and $(\mathrm{a}) \epsilon=0.05 \mathrm{w}_{0}$ and $(\mathrm{b}) \epsilon=$ $0.10 \mathrm{w}_{0}$. The smoothing of the edge of the beam profile dramatically decreases the oscillatory ringing in $\mathrm{F}_{1}$.

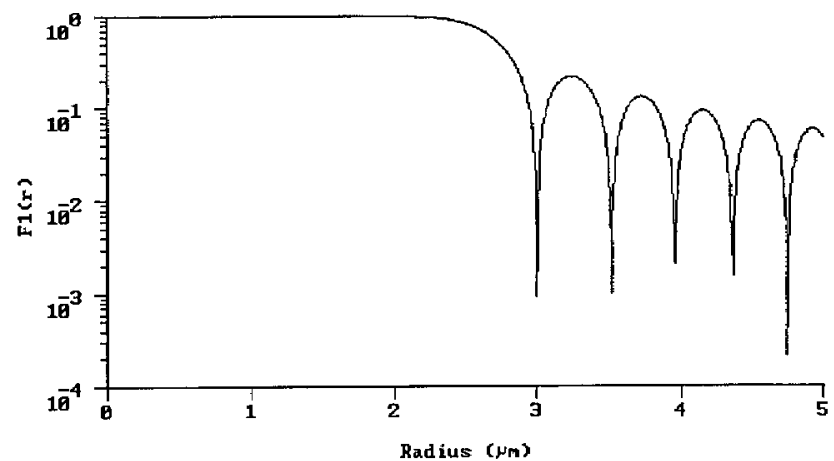

(c)

Fig. 5. Dominant beam-shape function $F_{1}$ for the localized tophat beam of Eq. (45) with (a) $\mathrm{w}_{0}=25 \mu \mathrm{m}$, (b) $\mathrm{w}_{0}=7.5 \mu \mathrm{m}$, and (c) $\mathrm{w}_{0}=2.5 \mu \mathrm{m}$ as a function of the distance $\rho$ from the $z$ axis.

new localized description of top-hat beams [eq. (51)], which improves on the description of Eq. (46) previously published in Ref. 10.

\section{Conclusion}

In the GLMT framework, the question what are the el ectromagnetic fields of a laser beam is equivalent to the question what are the values of the BSC's that describe the beam? By the use of a so-called s-expansion method, one may obtain the fields of standard beams associated with standard BSC's. We claim that these coefficients represent an ideal description of a Gaussian beam. Standard beams make possible the study of both mildly focused and extremely focused beams. The so-called localized approximation received a rigorous justification. It provides a simple analytical expression for the BSC's that is very close to standard beam values. Finally, the local ized interpretation of partial-wave expansions that underlies the localized approximation received further support from the investigation of top-hat beams, because they may beaccurately described with thelocalized approximation.

This work was supported in part by National Aeronautics and Space Administration grant NCC-3204.

\section{References}

1. G. Gréhan, G. Gouesbet, A. Naqwi, and F. Durst, "Particle trajectory effects in phase-Doppler systems: computations and experiments," Part. Part. Syst. Charact. 10, 332-338 (1993) 
2. G. Gréhan, G. Gouesbet, A. Naqwi, and F. Durst, "Trajectory ambiguities in phase-Doppler systems: study of a nearforward and a near-backward geometry," Part. Part. Syst. Charact. 11, 133-144 (1994).

3. S. A. Schaub, D. R. Alexander, and J . P. Barton, "Theoretical analysis of the effects of particle trajectory and structural resonances on the performance of a phase-Doppler particle analyzer,"Appl. Opt. 33, 473-483 (1994).

4. K. F. Ren, G. Gréhan, and G. Gouesbet, "Laser sheet scattering by spherical particles," Part. Part. Syst. Charact. 10, 146-151 (1993).

5. G. Gréhan, K. F. Ren, G. Gouesbet, A. Naqwi, and F. Durst, "Evaluation of a particle sizing technique based on laser sheets," Part. Part. Syst. Charact. 11, 101-106 (1994).

6. K. F. Ren, G. Gréhan, and G. Gouesbet, "Evaluation of laser-sheet beam shape coefficients in generalized Lorenz-Mie theory by using a localized approximation,"J . Opt. Soc. Am. A 11, 2072-2079 (1994).

7. D. Allano, G. Gouesbet, G. Gréhan, and D. Lisiecki, "Droplet sizing using a top-hat laser beam technique," J . Phys. D 17, 43-58 (1984).

8. G. Gréhan and G. Gouesbet, "Simultaneous measurements of velocities and sizes of particles in flows using a combined system incorporating a top-hat beam technique," Appl. Opt. 25, 3527-3538 (1986).

9. M. Maeda and K. Hishida, "Application of top-hat laser beam to particle sizing in LDV system," in Proceedings of the First International Symposium on Optical ParticleSizing: Theory and Practice(Plenum, New York, 1988).

10. F. Corbin, G. Gréhan, and G. Gouesbet, "Top-hat beam technique: improvements and application to bubble measurements," Part. Part. Syst. Charact. 8, 222-228 (1991).

11. G. Gréhan and G. Gouesbet, "Optical levitation of a single particle to study the theory of the quasi-elastic scattering of light,"Appl. Opt. 19, 2485-2487 (1980)

12. G. Gouesbet, B. Maheu, and G. Gréhan, "Light scattering from a sphere arbitrarily located in a Gaussian beam, using a Bromwich formulation," J . Opt. Soc. Am. A 5, 1427-1443 (1988).

13. J . P. Barton, D. R. Alexander, and S. A. Schaub, "Internal and near-surface electromagnetic fields for a spherical particle illuminated by a focused laser beam," J. Appl. Phys. 64, 1632-1639 (1988).

14. G. Gouesbet, G. Gréhan, and B. Maheu, "Generalized LorenzMie theory and applications to optical sizing," in N. Chigier ed., Combustion Measurements (Hemisphere, New York, 1991), pp. 339-384.
15. G. Gouesbet, "Generalized Lorenz-Mie theory and applications," Part. Part. Syst. Charact. 11, 22-34 (1994).

16. L. W. Davis, "Theory of electromagnetic beams," Phys. Rev. A 19, 1177-1179 (1979).

17. J. P. Barton and D. R. Alexander, "Fifth-order corrected electromagnetic field components for a fundamental Gaussian beam,"J . Appl. Phys. 66, 2800-2802 (1989).

18. G. Gouesbet, B. Maheu, and G. Gréhan, "The order of approximation in a theory of the scattering of a Gaussian beam by a Mie scatter center,"J . Opt. (Paris) 16, 239-247 (1985); republished in Selected Papers On Light Scattering, M. Kerker, ed., Vol. 951 of SPIE Milestone Series (Society of Photo-Optical Instrumentation Engineers, Bellingham, Wash., 1988), Part 1, pp. 352-360.

19. J. A. Lock and G. Gouesbet, "Rigorous justification of the localized approximation to the beam-shape coefficients in generalized Lorenz-Mie theory. I: On-axis beams," J . Opt. Soc. Am. A 11, 2503-2515 (1994).

20. G. Gréhan, B. Maheu, and G. Gouesbet, "Scattering of laser beams by Mie scatter centers: numerical results using a localized approximation,"Appl. Opt. 25, 3539-3548 (1986).

21. J . A. Lock, "Contribution of high-order rainbows to the scattering of a Gaussian laser beam by a spherical particle," J . Opt. Soc. Am. A 10, 693-706 (1993).

22. B. Maheu, G. Gréhan, and G. Gouesbet, "Ray localization in Gaussian beams," Opt. Commun. 70, 259-262 (1989).

23. H. C. van de Hulst, Light Scattering by Small Particles (Dover, New York, 1981), Sects. 12.31, 12.33.

24. G. Gouesbet, G. Gréhan, and B. Maheu, "Expressions to compute the coefficients $g_{n}{ }^{m}$ in the generalized Lorenz-Mie theory, using finite series,"J . Opt. (Paris) 19, 35-48 (1988).

25. G. Gouesbet, G. Gréhan, and B. Maheu, "Computations of the coefficients $g_{n}$ in the generalized Lorenz-Mie theory using three different methods,"Appl. Opt. 27, 4874-4883 (1988).

26. G. Gouesbet, G. Gréhan, and B. Maheu, "A localized interpretation to compute all the coefficients $g_{n} \mathrm{~m}$ in the generalized Lorenz-Mie theory,"J . Opt. Soc. Am. A 7, 998-1007 (1990).

27. G. Gouesbet and J. A. Lock, "Rigorous justification of the localized approximation to the beam-shape coefficients in generalized Lorenz-Mie theory. II: Off-axis beams," J . Opt. Soc. Am. A 11, 2516-2525 (1994).

28. S. A. Schaub, J. P. Barton, and D. R. Alexander, "Simplified scattering coefficients for a spherical particle located on the propagation axis of a fifth-order Gaussian beam," Appl. Phys. Lett. 55, 2709-2711 (1989).

29. G. Arfken, Mathematical Methods for Physicists, 3rd ed. (Academic, New York, 1985), Chap. 15. 